\title{
Anatomia interna de alguns moluscos bivalves fósseis do grupo Bauru (cretáceo superior, Bacia Bauru), Brasil: implicações paleoecológicas
}

\section{Internal anatomy of some bivalve mollusk fossils of the Bauru group (late cretaceous, Bauru Basin), Brazil: paleoecological implications}

\author{
Renato Pirani Ghilardi \\ Universidade Estadual Paulista - Bauru \\ Maria da Saudade Araujo Santos Maranhão Rosa \\ Instituto Geológico do Estado de São Paulo
}

\begin{abstract}
Resumo: Análises mais detalhadas em espécies de moluscos bivalves do Grupo Bauru (Cretáceo Superior, Bacia Bauru), depositadas na coleção científica Sérgio Mezzalira (IG/ SMA/SP), demonstram a preservação de feições musculares ainda não descritas para o táxon Anodontites nos estados de São Paulo e Minas Gerais. Tais feições, associadas à anatomia externa dos táxons, contribuem para o melhor entendimento do hábito de vida desses organismos dulciaquícolas que, provavelmente, estão intimamente relacionados às variações energéticas sazonais dos rios onde viviam. Apesar do registro esparso aqui notificado, as novas informações ampliam o panorama paleoecológico para os bivalves do Cretáceo Superior da Bacia Bauru.
\end{abstract}

Palavras-chave: Cretáceo superior. Bivalves fósseis. Bacia Bauru. Mutelidae.

Abstract: Detailed analyses in species of fossil bivalve mollusks of the Bauru Group (Late Cretaceous, Bauru Basin), exposed in the Sérgio Mezzarila scientific collection (IG/ SMA/SP), expose the preservation of musculature scars that have not been described as yet to Anodontites in São Paulo and Minas Gerais state. These features, which are associated to taxa-external anatomies, contribute to better understand the life habits of these freshwater organisms that were probably related to seasonal energetic variations in the rivers, where these organisms lived. Despite the fragmentary character of this record, the new information broadens the paleoecological panorama of bivalves in the Late Cretaceous of the Bauru Basin.

Keywords: Late Cretaceous. Fossil Bivalves. Bauru Basin. Mutelidae. 


\section{INTRODUÇÃO}

A Bacia do Paraná abrange os territórios dos estados de Mato Grosso, Paraná, São Paulo e partes dos territórios dos estados de Minas Gerais e Goiás, estendendo-se pelo Brasil, Uruguai, Paraguai e Argentina. É uma bacia sedimentar intracratônica (sinéclise), que evoluiu sobre a Plataforma Sul-Americana, e sua formação teve início há cerca de 400 milhões de anos, no Período Devoniano, terminando no Cretáceo.

Sob a Bacia do Paraná deposita-se a Bacia do Bauru (Figura 1), que é a mais extensa sequência sedimentar de idade cretácea da América do Sul. Sua constituição é de arenitos e siltitos depositados em ambiente fluvial. É nesse ambiente que encontramos o Grupo Bauru, que pode ser dividido em três intervalos estratigráficos: a) intervalo inferior, assentado sobre um substrato basáltico irregular; b) intervalo médio, compreendendo sedimentos essencialmente arenosos de origem aluvial; e c) intervalo superior, englobando na base um componente lacustre que passa, vertical e lateralmente para oeste, a depósitos aluviais arenosos. (CASTRO et al., 1999). O Grupo Bauru cobre todo o planalto do estado de São Paulo, estende-se ao extremo oeste de Minas Gerais, ao sul de Goiás e ao sul e leste de Mato Grosso, sendo sua estratigrafia e idade temas de grande controvérsia na literatura. (Cf. BATEZELLI et al., 2007).

Dentre os fósseis do Grupo Bauru (Cretáceo Superior, Bacia Bauru), certamente os mais conhecidos são os répteis crocodilianos, quelônios e dinossaurídeos. Contudo, outros macrofósseis podem ser descritos para esses sedimentos, como vegetais, ostrácodes, conchostráceos e restos de peixes. O grupo dos moluscos dulciaquícolas é o menos estudado do Grupo Bauru, apesar de o táxon ser considerado como importante ferramenta de refinamento em interpretações paleoambientais.

\section{BREVE HISTÓRICO DAS PES- QUISAS SOBRE MOLUSCOS DO GRUPO BAURU}

Ihering (1913) foi o primeiro a descrever um bivalve para os sedimentos do Grupo Bauru. A espécie Itaimbea priscus (IHERING, 1913) foi descrita em sedimentos no município de São José dos Dourados, estado de São Paulo. Apenas cerca de 50 anos posteriormente a essa descrição, Arid et al. (1962) descrevem um gastrópode em afloramentos nas imediações de São José do Rio Preto. Mezzalira (1966), Suarez e Arruda (1968) e Parodiz (1969) aumentam o número de espécies descritas de moluscos para o estado de São Paulo, porém sem classificá-las taxonomicamente de maneira precisa.

O trabalho de Mezzalira (1974) é um marco para o estudo desses moluscos fósseis. $\mathrm{O}$ autor descreve a maior quantidade de táxons conhecidos (?Hydrobia prudentinensis, Viviparus souzai, Physa aridi, Diplodon arrudai, Florenceia peiropolensis, Sancticarolis tolentinoi, Taxodontites paulistanensis, Anodontites freitasi, Anondotites pricei e Monocondylaea cominatoi), além de organizar sistematicamente os moluscos do Grupo Bauru na maneira atualmente empregada. Adicionalmente, Mezzalira (1974) descreve de modo pioneiro esses animais em sedimentos de Goiás e Minas Gerais.

Simone e Mezzalira (1993) voltam ao tema ao descrever a preservação de restos de partes moles (palpos, demibrânquias e músculos) em um bivalve Unionoida (Anodontites freitasi) do Grupo Bauru, no estado de São Paulo. Campanha et al. (1993) descrevem o gastrópode ?Hydrobya sp. e Turritella sp. em sedimentos localizados entre Uberaba e Uberlândia, MG. 


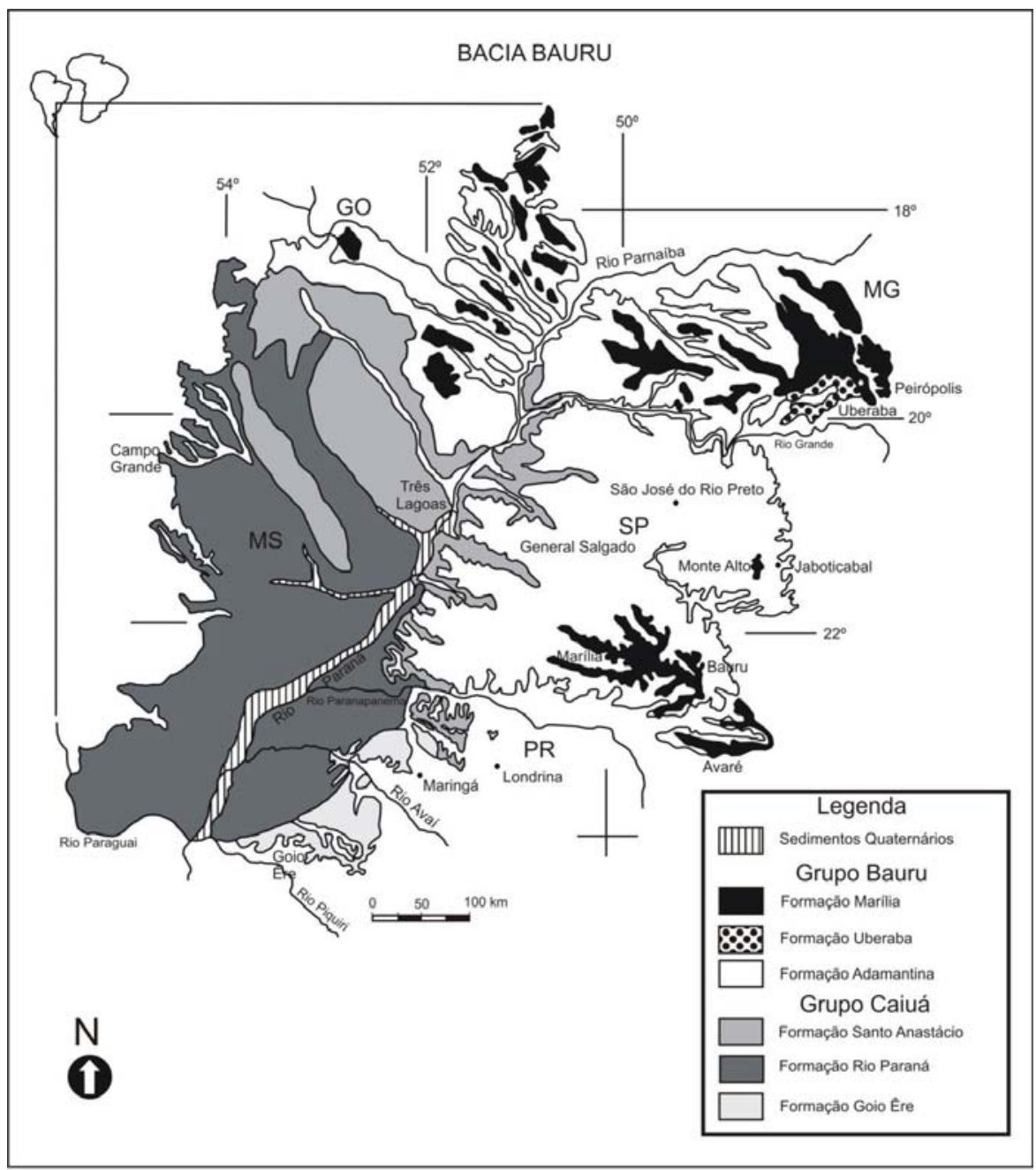

Figura 1 - Mapa litoestratigráfico da parte oriental da Bacia Bauru.

Fonte: modificado de MARINHO et AL. (2006).

Senra e Silva (1999) relatam a ocorrência de microbivalves em sedimentos de Uberaba, MG. As autoras classificam provisoriamente esse novo táxon como ?Musculium. Mezzalira e Simone (1997) normalizam a situação taxonômica dos gêneros Florenceia, Sancticarolis e Taxodontites já alterados previamente, mas sem valor taxonômico, em Simone e Mezzalira (1994). Finalmente, Mezzalira e Simone (1999) descrevem, a partir de material depositado em coleção científica, o gênero Castalia para sedimentos do Grupo Bauru sendo constituído por duas espécies (C. cretacea e C. minuta).

É notória a carência de trabalhos paleoecológicos e tafonômicos dos bivalves e gastrópodes fósseis do Cretáceo Superior da Bacia Bauru. Recentemente, entretanto, alguns resumos apresentados em congressos tramam considerações mais modernas sobre os moluscos (GHILARDI; BENEDETTI, 2004; GHILARDI et al., 2006; GHILARDI et al., 2007; D'AGOSTA; GHILARDI, 2008; CARBONARO et al., 2009), demonstrando a viabilidade do trabalho com esse táxon e a possibilidade de interpretações paleoambientais mais precisas.

Adicionalmente, a paleofauna de invertebrados representada no Grupo Bauru apresenta algumas semelhanças com os depósitos argentinos do Cretáceo Superior. Inclusive, algumas variedades já eram conhecidas na Argentina, como o gênero Physa. (MARTINELLI et al., 2007). Entretanto, a presença de formas exclusivas sugere que pode ter ocorrido algum endemismo na fauna. Uma das hipóteses para a existência de espécies particulares era a diferenciação climática do Grupo Bauru, mais seco, provocando um "stress" ambiental e gerando mudanças particulares nos animais.

\section{PALEONTOLOGIA SISTEMÁTICA}

\section{Classe Bivalvia}

Subclasse Palaeoheterodonta Newell, 1965

Ordem Unionoida Stoliczka, 1871

Superfamília Unionacea Fleming, 1828

Família Mutelidae Swainson, 1840

Gênero Anodontites Bruguiere, 1792 
Diagnose genérica: concha arredondada a elongada, inflada, moderadamente espessa; umbo presente e liso; perióstraco liso ou entrelaçado raramente raiado; charneira edentelosa com a linha de charneira reta ou tenuamente curvada; lúnula distinta e grande.

\section{Anodontites pricei Mezzalira, 1974}

Diagnose específica: concha elítica; umbo baixo, liso, ocupando a posição mais próxima da borda anterior; inequilátera. Borda dorsal subreta; borda ventral ligeiramente abaulada; borda posterior subreta e borda anterior convexa. Superfície com finas linhas concêntricas de crescimento. Charneira aparentemente edentelosa.

Tipos: Holótipo 738-I (Coleção Científica Sérgio Mezzalira/Instituto Geológico da Secretaria do Meio Ambiente do Estado de São Paulo). Molde interno de valva direita. Parátipo 739-I: molde interno de valva direita.

Localidade-tipo e estratigrafia: Peirópolis, MG. Contexto estratigráfico: Bacia Bauru, Formação Marília, Cretáceo Superior.

Ementa específica: No parátipo de Anodontites pricei, a cicatriz muscular adutora anterior é grande, circular e sem estrias. A cicatriz retratora anterior é bem definida e ligada à porção dorsal do adutor (Figura 2). Musculatura posterior adutora também é grande e circular (Figura 2). A musculatura retratora posterior não é preservada. A linha palial é bem definida, integropaliada e larga ligando as ventrais da musculatura isomiária.

Comentário: Apesar de Mezzalira (1974) descrever musculatura adutora anterior a esse espécime, não houve representação satisfatória da mesma.

\section{? Anodontites freitasi Mezzalira, 1974}

Diagnose específica: concha subelítica, estreita e alongada; inequilátera e equivalva. Umbo baixo, liso; bico liso, raso, subanterior. Depressão mediana acentu- ada na porção inferior da concha. Borda dorsal levemente angulosa com extensão posterior pouco maior que a anterior; borda ventral subreta e levemente convexa. Com suave concavidade na sua extensão medioposterior, passando em curva continua para a borda anterior. Esta é curta com convexidade acentuada. Borda posterior, a julgar pelas linhas de crescimento, curta subvertical passando em curva forte na borda ventral. Linhas de crescimento aparentemente subconcêntricas mais ou menos acentuadas.

Tipos: Holótipo 742-I (Coleção Científica Sérgio Mezzalira do Instituto Geológico da Secretaria do Meio Ambiente do Estado de São Paulo). Contra moldes de valvas conjugadas.

Localidade-tipo e estratigrafia: Pacaembu Paulista, SP. Contexto estratigráfico: Bacia Bauru, Formação Adamantina, Cretáceo Superior.

Ementa específica: Em ?Anodontites freitasi, a cicatriz muscular adutora anterior é pequena, subcircular e sem estrias (Figura 2). Há um provável músculo retrator anterior ligado à porção dorsal do adutor. A musculatura posterior não é definida em nenhum exemplar, mas provavelmente constitui-se de forma isomiária. A linha palial é simples, relativamente larga e sem estrias em sua porção anterior.

Comentário: Simone e Mezzalira (1993) descrevem, para outro espécime depositado na Coleção Científica Sérgio Mezzalira, a presença de inserções na concha de partes moles (brânquias, palpos e músculos) na espécie. Contudo, a informação não foi utilizada para incrementar sua diagnose da espécie. 


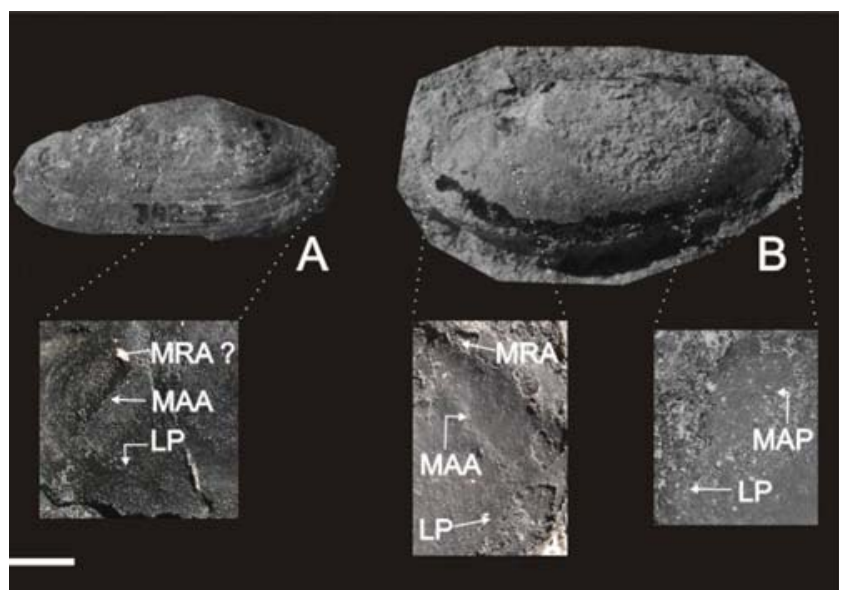

Figura 2 - Cicatrizes da musculatura de?Anodontites freitasi (742-I, A) e Anodontites pricei (739-I, B). Escala válida somente para as conchas $=1 \mathrm{~cm}$. Nota: LP = Linha Palial, MAA = Músculo Adutor Anterior, MRA = Músculo Retrator Anterior, MAP = Músculo Adutor Posterior.

Org: GHILARDI, 2010

\section{DISCUSSÃO}

Os estudos de bivalves de água doce nunca receberam a mesma atenção dispensada ao estudo de formas marinhas. Entretanto, algumas características morfológicas destes últimos são exclusivas do grupo. (Cf. GHILARDI; SIMÕES, 2002, para síntese). A carência de estudos se deve, aparentemente, às poucas adaptações inerentes ao grupo dulcícola, assim como seus padrões evolutivos pouco complexos. (SAVAZZI; PEIYI, 1992).

Adicionalmente, o caráter continental - com fácies terrígena e lacustre -, que lhe permite uma descontinuidade na sedimentação dos seus termos litológicos e lhe empresta distribuição lenticular (MEZZALIRA, 1974), é um dos principais fatores que alimenta as diferentes opiniões a respeito de sua estratigrafia e, consequentemente, ambientes de deposição. A Formação Adamantina teria sistemas deposicionais mais energéticos, sendo representada por sedimentos relativos a rios de grande porte. Ainda nessa formação encontram-se sedimentos representativos de ambientes de planícies de inundação, associados a sedimentos de canais fluviais. O clima seria quente e úmido, alternado por períodos mais secos. Já a Formação Marília reflete o ambiente fluvial meandrante progressivamente sendo alterado para um sistema fluvial anastomosado, devido ao aumento da aridez e desertificação.

Algumas considerações ecológicas podem ser elaboradas em relação aos bivalves Anodontites do Grupo Bauru. A morfologia externa dos exemplares revela índices médios de obesidade (altura/largura) próximos a 2,0 e índices médios de alongamento (comprimento/altura) próximos a 1,4 - o que configura forma comprimida e alongada às conchas analisadas. (STANLEY, 1970). A forma laminar desses organismos, assim, está relacionada ao modo de vida em substratos estáveis, com possibilidade de rápida reescavação quando da exumação das conchas. (Ibidem). Esse hábito é compatível com a provável presença de alta energia e sazonalidade dos rios, conforme demonstra a sedimentologia. Contudo, é necessário ressaltar que o hábito de reescavação de moluscos bivalves de água doce ainda não é bem descrito na literatura e a maioria dos grupos recentes não possuem esse comportamento. (GRAY, 2001).

Adicionalmente, a forma da concha, mesmo com a ausência do sinus palial preservado, indica um hábito escavador intermediário a profundo para os organismos. Esse fato é corroborado pela não preservação de injúrias ou marcas de predação nas conchas analisadas. De fato, bivalves que se soterram mais profundamente ao substrato tendem a ter menor ação de organismos muluscívoros. (STANLEY, 1970; SAVAZZI; PEIYI, 1992). 


\section{CONCLUSÃO}

A descrição e análise da anatomia interna dos bivalves do Grupo Bauru é rara na literatura. (Cf. SIMONE; MEZZALIRA 1993, para uma bela exceção). De fato, cicatrizes musculares e dentição são características ausentes na descrição das espécies desses moluscos.

Entretanto, a análise pormenorizada dos bivalves das coleções científicas permitiu o reconhecimento de algumas feições anatômicas, particularmente no que se refere ao gênero Anodontites. Essas feições podem vir a alterar alguns conceitos sobre a sistemática desses organismos com inferências sobre a paleoecologia e tafonomia do grupo. De fato, os moluscos do Grupo Bauru reservam ainda muitas informações para o melhor entendimento do paleoambiente do Cretáceo Superior da Bacia Bauru.

\section{AGRADECIMENTOS}

Os autores gostariam de agradecer a Tiossi, A. B., Zanzini, I. H. e Carbonaro, F. A. pela ajuda nos trabalhos de coleta de material e de preparo das amostras. Pesquisa realizada com apoio financeiro da FAPESP (Proc. 05/00506-5).

\section{REFERÊNCIAS}

ARID, F. M.; VIZZOTO, L. P.; LANDIN, P. M. P. A ocorrência de um jazigo fossilífero nos arredores de São José do Rio Preto. Ciência e Cultura, n. 14, v. 3, p. 172, 1962.

BATEZELLI, A.; SAAD, A. R.; BASILICI, G. Arquitetura deposicional e evolução da seqüência aluvial neocretácea da porção setentrional da Bacia Bauru, no sudeste brasileiro. Revista Brasileira de Geociências, p. 44-54, 2007.

CASTRO, J. C.; DIAS-BRITO, D.; MUSACCHIO, E.; SUAREZ, J.; MARANHÃO, M. S. A. S.; \& RODRI-
GUEZ, R. Arcabouço estratigráfico do Grupo Bauru no oeste paulista, Brasil. Resumos Simpósio sobre o Cretáceo do Brasil, 5 Simpósio sobre el Cretáceo de América del Sur, 1. Serra Negra - SP, 1999

CAMPANHA, V. A.; ETCHEBEHERE, M. L. C.; SAAD, A. R.; FÚLFARO, V. J. Novas ocorrências fossilíferas no Grupo Bauru na região do triângulo mineiro, MG. Geociências. n. 12, v. 2, p. 353-372, 1993.

CARBONARO, F. A.; GHILARDI, R. P.; NAVA, W. Descrição de Physidae do Cretáceo superior (formação Adamantina, Bacia Bauru) na região do município de Marília, SP. Resumos Paleo 2009. Núcleo São Paulo da Sociedade Brasileira de Paleontologia, Guarulhos, p. 33, 2009.

D’AGOSTA, F. C. P.; GHILARDI, R. P. Bivalves Fósseis do Grupo Bauru: Estado Atual do Conhecimento. In: CONGRESSO BRASILEIRO DE ZOOLOGIA, 27. Sociedade Brasileira de Zoologia, Curitiba, 2008. Anais...

GHILARDI, R. P.; SIMÕES, M. G. Foram os bivalves do Grupo Passa Dois (exclusive formação Rio do Rasto), Neopermiano, invertebrados tipicamente Dulcícolas? Pesquisa em Geociências, n. 29, v. 1, p. 91-102, 2002.

GHILARDI, R. P.; BENEDETTI, G. F. S. Reconhecimento de Cicatrizes Musculares e Considerações Prévias sobre a Paleoecologia e Tafonomia de ?Anodontites (Bivalvia, Unionoida) do Grupo Bauru (Cretáceo Superior, Bacia Bauru). Resumos Paleo 2004. Núcleo São Paulo da Sociedade Brasileira de Paleontologia, São Carlos, p. 12-13, 2004.

GHILARDI, R. P.; D'AGOSTA, F. C. P.; TAVARES, S. S.; CAMPOS, A. C. A. Taphonomy of late Cretaceous freshwater bivalves from Bauru Group, Bauru Basin (Monte Alto County, São Paulo State, Brazil): paleoenvironmental implications. Scientific Program and Abstractrs of International Congress on Bivalvia - Bivalvia 2006. Barcelona, p. 37, 2006.

GHILARDI, R. P.; D’AGOSTA, F. C. P.; SIMONE, L. R. L. Itaimbea sp. nov., uma nova espécie de Iridinidae fóssil (Bivalvia: Etherioidea) do Grupo Bauru (Bacia Bauru, Cretáceo Superior), Monte Alto, São Paulo, Brasil. In: CONGRESSO BRASILEIRO DE PALEONTOLOGIA, 20. Anais... Sociedade Brasileira de Paleontologia, Búzios - RJ, p. 170, 2007.

GRAY, John S. Marine diversity: the paradigms in patterns of species richness examined. Sci Mar. n. 65, 41-56, 2001. 
IHERING, H. Pleiodon priscus. In: COMISSÃO GEOGRÁPHICA E GEOLÓGICA 1913. São José dos Dourados, São Paulo: Exploração do Rio Grande e seus afluentes. São José dos Dourados, p. 39, 1913.

MARINHO, T. S.; RIBEIRO, L. C. B., CARVALHO, I. S. Morfologia de osteodermos de Crocodilomorfos do Sítio Paleontológico de Peirópolis (Bacia Bauru, Cretáceo Superior). Anuário do Instituto de Geociências, Rio de Janeiro, v. 29, p. 42-51, 2006.

MARTINELLI, A. G.; GARRIDO, A. C.; FORASIEPI, A. M. ; PAZ, E. R. E GUROVICH, Y. Notes on fossil remains from the Early Cretaceous Lohan Cura Formation, Neuquén Province, Argentina. Gondwana Research, n. 11, p. 537-552, 2007.

MEZZALIRA, S. Os fósseis do Estado de São Paulo. Boletim do Instituto Geográfico e Geológico, 1966.

MEZZALIRA, S. Contribuição ao conhecimento da estratigrafia e paleontologia do arenito Bauru. Boletim do Instituto Geográfico e Geológico, $\mathrm{n}$. 51, p. 1-163, 1974.

MEZZALIRA, S.; SIMONE, L. R. L. A posição sistemática de alguns bivalves Unionoidea do grupo Bauru (Cretáceo Superior) do Brasil. Geociências UNG, n. 2, v. 6, p. 63-65, 1997.

Duas novas espécies de Castalia (Mollusca, Bivalvia, Unionoida) no Cretáceo do Grupo Bauru, São Paulo, Brasil. Geociências, n. 4, p. 58-60, 1999.

PARODIZ, J. J. The Tertiary Non-Marine Mollusca of South America. Annals of Carnegie Museum, n. 40, p. $1-242,1969$.

SAVAZZI, E.; PEIYI, Y. Some morphological adaptations in freshwater bivalves. Lethaia, n. 25, p. 195-209, 1992.

SENRA, M. C. E.; SILVA, L. H. S. Moluscos dulçaquícolas e microfósseis vegetais associados da Formação Marília, Bacia Bauru (Cretáceo Superior), Minas Gerais, Brasil. Boletim do $5^{\circ}$ Simpósio sobre o Cretáceo do Brasil, p. 497-500, 1999.

SIMONE, L. R. L.; MEZZALIRA, S. Vestígios de partes moles em um bivalve fóssil (Unionoida, Mycetopodidae) do Grupo Bauru (Cretáceo Superior), São Paulo, Brasil. Anais da Academia Brasileira de Ciências, n. 65, p. 155-159, 1993.

Fossil Molluscs of Brazil. Boletim do Instituto Geológico, n. 11, p. 1-202, 1994.
STANLEY, S. M. Relation of shell form to life habits of the Bivalvia (Mollusca). Geological Society of America, Memoirs, n. 125, p. 1-296, 1970.

SUAREZ, J. M.; ARRUDA, M. R. Jazigo fossilífero no Grupo Bauru, contendo lamelibrânquios. In: CONGRESSO BRASILEIRO DE GEOLOGIA, 22., Sociedade Brasileira de Geologia, Belo Horizonte, Anais... p. 209-212, 1968.

Recebido em 08/06/10

Aceito para publicação em 14/07/10 


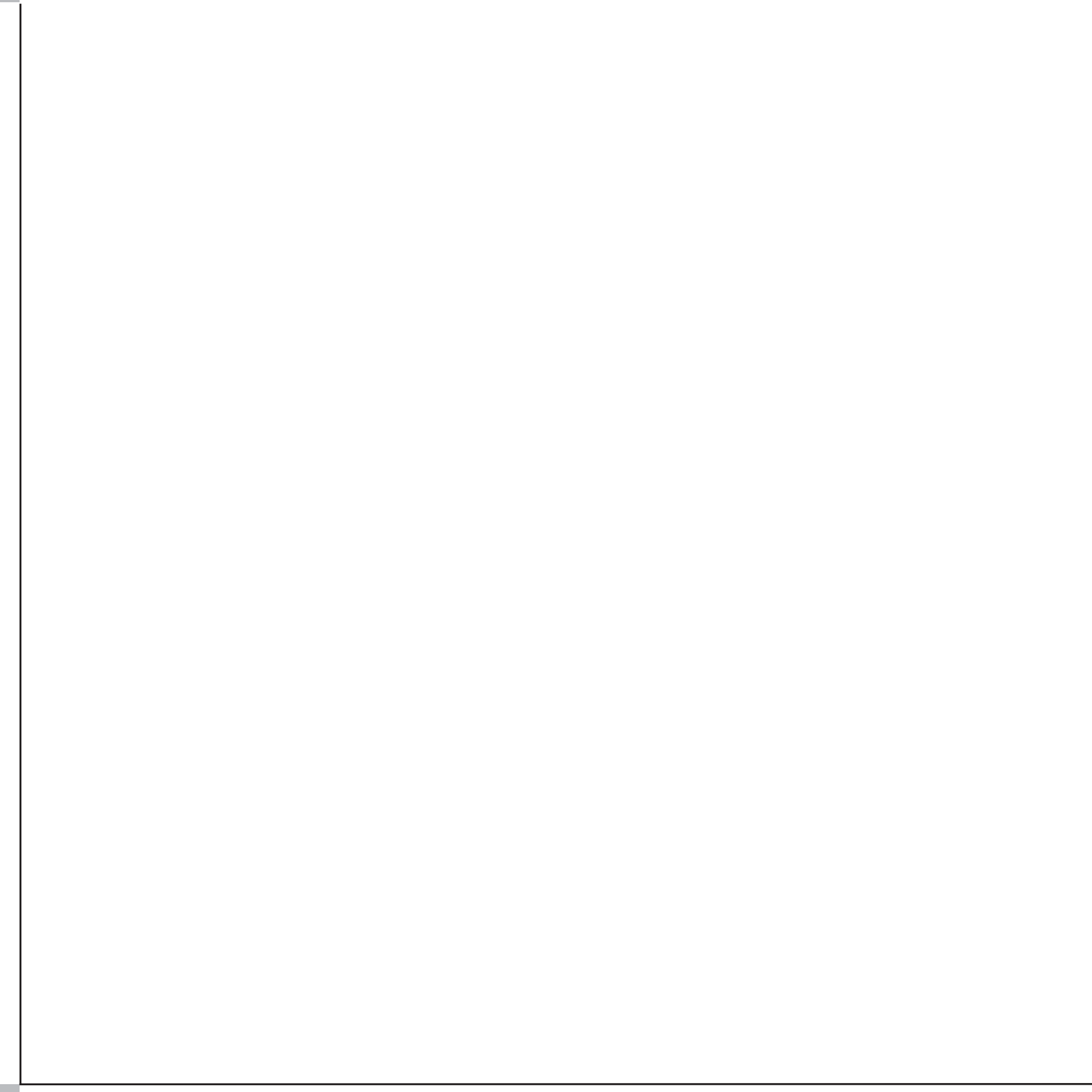

